\title{
TNFAIP2 expression induces epithelial-to-mesenchymal transition and confers platinum resistance in urothelial cancer cells
}

\author{
Naoya Niwa ${ }^{1} \cdot$ Nobuyuki Tanaka $\mathbb{1}^{1} \cdot$ Hiroshi Hongo ${ }^{1} \cdot$ Yasumasa Miyazaki $^{1} \cdot$ Kimiharu Takamatsu $^{1} \cdot$ \\ Ryuichi Mizuno ${ }^{1} \cdot$ Eiji Kikuchi $^{1} \cdot$ Shuji Mikami ${ }^{2}$. Takeo Kosaka $\mathbb{D}^{1} \cdot$ Mototsugu Oya $^{1}$
}

Received: 13 September 2018 / Revised: 2 May 2019 / Accepted: 7 May 2019 / Published online: 1 July 2019

(c) The Author(s), under exclusive licence to United States and Canadian Academy of Pathology 2019

\begin{abstract}
Cisplatin (CDDP)-based chemotherapy is the gold standard treatment for many types of cancer. However, the phenotypic hallmark of tumors often changes after CDDP treatment, with the acquisition of epithelial-to-mesenchymal transition (EMT) and platinum resistance. Furthermore, the mechanisms by which cancer cells acquire EMT under the control of CDDP remain unclear. Following an investigation of urothelial carcinoma (UC) before and after the acquisition of platinum resistance, we offer the new target TNFAIP2, which led to EMT and tumor invasion in platinum-treated UC cells. TNFAIP2 expression in cancer was examined at the protein and transcriptional levels. A potential target for TNFAIP2 during EMT was assessed by microarray. Clinically, upregulated TNFAIP2 expression was identified as a significant predictor of mortality following surgery in three different cohorts of patients with UC $(n=156, n=119$, and $n=54)$. Knockdown of TNFAIP2 resulted in upregulation of E-cadherin expression and downregulation of TWIST1 expression, which decreased motile function in platinum-resistant UC cells. TNFAIP2 overexpression led to downregulation of E-cadherin expression and upregulation of TWIST1 expression in platinum-naïve UC cells. Clinical investigation of matched pre- and post-CDDPtreated UC sections confirmed upregulation of TNFAIP2 expression in CDDP-treated tumors but downregulation of Ecadherin expression. Global gene expression analysis following TNFAIP2 knockdown identified MTDH as a positive regulator of TNFAIP2-derived EMT acquisition in cancer cells. The present results suggest a relationship between TNFAIP2 and EMT in cancers under the control of CDDP, in which MTDH expression levels in cancer cells are vital for promoting TNFAIP2-derived EMT acquisition.
\end{abstract}

These authors contributed equally: Naoya Niwa, Nobuyuki Tanaka

Supplementary information The online version of this article (https:// doi.org/10.1038/s41374-019-0285-y) contains supplementary material, which is available to authorized users.

Nobuyuki Tanaka

urotanaka@keio.jp

$\triangle$ Takeo Kosaka

takemduro@gmail.com

$\triangle$ Mototsugu Oya moto-oya@keio.jp

1 Department of Urology, Keio University School of Medicine, Tokyo 160-8582, Japan

2 Department of Diagnostic Pathology, Keio University Hospital, Tokyo 160-8582, Japan

\section{Introduction}

Urothelial carcinoma (UC) is a primary tumor arising in the urothelium of the renal pelvis, ureter (the so-called upper urinary tract), and urinary bladder [1, 2]. Although organconfined UC may be surgically resected, the prognosis of locally invasive and/or metastatic disease remains poor [3]. Patients with metastatic or recurrent disease after radical surgery routinely receive cisplatin-based (CDDP-based) chemotherapy, such as M-VAC (methotrexate, vinblastine, doxorubicin, and CDDP) [4] and/or GC (gemcitabine and CDDP) regimens [5]. Since chemotherapy for UC has limited efficacy, most tumors relapse and eventually develop resistance to platinum.

Epithelial-to-mesenchymal transition (EMT) is a process by which epithelial cells lose their cell polarity and cell-to-cell adhesion and gain migratory and invasive potentials to become mesenchymal cells. The loss of Ecadherin expression is considered to be a key step in the 
initiation of EMT and is mediated by several transcriptional factors, such as TWIST1, SNAIL, SLUG, and ZEB1/2 [6, 7]. Accumulating evidence has demonstrated the acquisition of EMT in cancer cells under the control of CDDP $[8,9]$. However, limited information is currently available on the underlying molecular mechanisms.

We previously established UC cell lines with acquired platinum resistance, 5637PR, which were derived from the platinum-naïve UC cell line 5637 [10]. We reported that these cells had a robust phenotype and underwent EMT [11]. Marked differences were observed in migratory potential before and after the acquisition of platinum resistance, and we demonstrated that ubiquitinproteasome dysregulation may play a role in EMT when tumors acquire platinum resistance [11]. In this study, we investigated other mechanisms for the initiation of EMT in UC cells. We found that TNFAIP2 expression increased in human UC cells that acquired platinum resistance. TNFAIP2, a protein induced by TNF-alpha, is associated with tumor migration and invasion in other cancers [12-14], and we hypothesize that upregulated TNFAIP2 expression induces EMT in and confers platinum resistance to UC cells.

\section{Methods}

\section{Cell lines and culture conditions}

The human bladder cancer cell lines 5637, T24, and UMUC-3 were obtained from the American Type Culture Collection. Cells were routinely maintained in RPMI1640 (Invitrogen, Carlsbad, CA) supplemented with 10\% fetal bovine serum (FBS) (Dainippon Pharmaceutical, Tokyo, Japan) at $37^{\circ} \mathrm{C}$ in a humidified $5 \% \mathrm{CO}_{2}$ atmosphere. The acquired platinum resistance subline of 5637 cells, namely 5637PR, was previously established [10] and used in this study. Briefly, 5637 cells were grown in RPMI-1640 supplemented with 10\% FBS containing up to $3 \mu \mathrm{M} \mathrm{CDDP}$ at $37^{\circ} \mathrm{C}$ in a humidified $5 \% \mathrm{CO}_{2}$ atmosphere.

\section{Drugs and antibodies}

CDDP was generously supplied by Nippon Kayaku Co. (Tokyo, Japan) or purchased from Wako Pure Chemical Co. (Osaka, Japan). The monoclonal antibody for TNFAIP2 (\#sc28318) was purchased from Santa Cruz Biotechnology Inc. (Santa Cruz, CA). The monoclonal antibody for E-cadherin (\#ab1416) was purchased from Abcam Inc. (Cambridge, MA). The monoclonal antibody for Beta-actin (\#A5316) was purchased from SigmaAldrich (St. Louis, MO).

\section{Construction of TNFAIP2 expression vectors and transfection}

Human TNFAIP2 cDNA (4180 bp) was synthesized with reference to the human TNFAIP2 sequence registered in the Genebank Database (http://www.ncbi.nlm.gov/genbank/), accession number NM_006291, and then subcloned into the BamHI-XbaI sites of the pABpo-CMV Neo Vector (Takara Bio, Otsu, Japan). The TNFAIP2 expression vector or control empty vector was transfected into 5637 cells using TranaIT-2020 transfection Reagent (Mirus Bio, Madison, WI) according to the manufacturer's instructions. After $48 \mathrm{~h}$, cells were used for further experimentation.

\section{Cell extracts and WB analysis}

Whole-cell extracts were obtained using RIPA buffer (Cell Signaling Technology Inc., Danvers, MA) containing a protease inhibitor, according to the manufacturer's protocol. The extracted whole protein $(50 \mu \mathrm{g})$ with sample buffer containing 2-mercaptoethanol (Nacalai Tesque Inc., Kyoto, Japan) was separated on 4-15\% SDS-PAGE, transferred to a nitrocellulose membrane (Bio-Rad Laboratories, Hercules, $\mathrm{CA}$ ), and then incubated overnight with 5\% skim milk. Primary antibodies were applied at room temperature for $1 \mathrm{~h}$. The membranes were then incubated with the appropriate secondary antibodies. Signals were detected using an enhanced chemiluminescence reagent (ECL plus Western Blotting Detection System; Amersham Pharmacia Biotech, Amersham, UK) and analyzed. Signal intensity was qualified using an LAS3000 Images Analysis System (Fuji Film, Tokyo, Japan). Beta-actin was used as the internal protein control.

\section{Real-time PCR}

Total RNA from cultured cells was isolated using RNAiso Plus (Takara Bio) according to the manufacturer's protocol. RNA quantity and quality were evaluated using a Nanodrop ND 2000 spectrophotometer (Thermo Scientific, Waltham, MA). The reverse transcription of RNA to cDNA was performed using a High Capacity cDNA Archive Kit (Applied Biosystems, Foster City, CA). Quantitative gene expression was performed for TNFAIP2 (Hs_00196800), MTDH (HS_00757841), E-cadherin (Hs_00170423), SLUG (Hs_00950344), ZEBI (Hs_01566407), TWISTI (HS_00361186), and GAPDH (Hs_99999905) with genespecific probes (Applied Biosystems) using the Taqman Universal PCR Master Mix and 7500 Fast Real-time PCR system (Applied Biosystems). The protocol of cycling conditions was described previously [15]. Data were then quantified using the comparative $\mathrm{Ct}$ method for relative gene expression using GAPDH as an endogenous control. 


\section{Immunofluorescence analysis}

To evaluate immunofluorescence staining, $4 \times 10^{4}$ cells were seeded on 14-mm coverslips in 8-well plates (Thermo Fisher Scientific). After $24 \mathrm{~h}$, cells were washed with PBS, fixed in $4 \%$ paraformaldehyde, and permeabilized in cold PBS with $0.2 \%$ Triton X-100 at room temperature. After blocking, slides were incubated with the primary antibody for TNFAIP2 at room temperature for $1 \mathrm{~h}$. Slides were then incubated with an Alexa 555-conjugated secondary antibody. Coverslips were mounted on glass slides in Vectashield ${ }^{\circ}$ mounting medium containing DAPI, and visualized under a fluorescence microscope (IX8, Olympus, Tokyo, Japan).

\section{In vitro cell invasion assay}

The invasion of tumor cells was assessed by counting the number of cells that migrated through transwell inserts with a polyethylene terephthalate membrane (pore size of $8 \mu \mathrm{m}$ ) coated with a uniform layer of BD Matrigel Basement Membrane Matrix (BD Biosciences, San Jose, CA). Cells were seeded at a density of $5 \times 10^{4}$ per insert. After an incubation in RPMI 1640 medium with $1 \%$ FBS, cells that remained inside the inserts were removed, and those that invaded the reverse side of the inserts were rinsed and counterstained using a Diff-Quick stain Kit (Sysmex Co., Kakogawa, Japan). Cells that had migrated through the membranes were counted.

\section{Small interfering RNA}

TNFAIP2 expression was transiently downregulated using the following predesigned duplex siRNA directed against TNFAIP2 (si-TNFAIP2 A and B) and MTDH (si-MTDH A and $\mathrm{B}$ ). The sense sequences of siRNA for TNFAIP2 were as follows: si-TNFAIP2 A, 5'-GCAAGCAGCUGACGAA UUATT-3'; si-TNFAIP2 B, 5'-CAAACUUCGUGGAUCA AAATT-3; si-MTDH A, 5'-CCGAAGUACUCGUCA AAAATT-3'; si- $M T D H$ B, 5'-GAAUCUCCCAAACAAA UAATT-3'. 5637PR cells were transiently transfected with $10 \mathrm{nM}$ of si-TNFAIP2 A, si-TNFAIP2 B, si-MTDH A, or si$M T D H$ B using Lipofectamine 2000 (Invitrogen) in culture medium with RPMI 1640 only. After an incubation for $12 \mathrm{~h}$, siRNA was removed by replacing culture medium with RPMI 1640 with $10 \%$ FBS and cells cultured for $24 \mathrm{~h}$ were then used for further experiments. A mock-transfection control was prepared using the transfection reagent only.

\section{Patient selection and preparation}

All human studies were reviewed and approved by the Institutional Review Board, Keio University Hospital. Surgical specimens from urinary urothelial cancer patients who had been treated surgically for upper urinary tract $(n=$ $156)$ and urinary bladder $(n=70)$ tumors at our institution were used. No patients received prior radiotherapy before surgery. Among 70 patients with bladder cancer, 16 received neoadjuvant CDDP-based chemotherapy, while 54 did not. The main surgical procedure was radical nephroureterectomy for upper urinary tract cancer and cystectomy for bladder cancer. The dissection of regional lymph nodes was performed according to the attending physician's discrimination. All tumors were histologically confirmed to be UC. Tumors were staged according to the 2002 TMN classification and graded according to the 2004 WHO classification. Patients were generally followed up every 3-4 months for 2 years following surgery, every 6 months for the next 3 years, and then every 6-12 months thereafter. Disease recurrence was defined as any documented recurrence by radiographically or pathologically proven failure, including local and distant sites. The cause of death was established by the attending physician, a chart review corroborated by death certificates, or by death certificates alone. The human tissue array slide for 119 upper urinary tract tumors was obtained from US Biomax Inc. (Rockville, MD) with the ethical approval of the host company (catalog \#HRaU-Uro120Sur-01).

\section{Immunohistochemistry}

Formalin-fixed paraffin-embedded sections $(4-5 \mu \mathrm{m})$ and paraffin sections of 119 upper urinary tract urothelial cancer specimens (catalog \#HRaU-Uro120Sur-01, US Biomax Inc.) were deparaffinized, rehydrated, and washed in PBS. Endogenous peroxidase was quenched and sections were blocked with skim milk. Primary antibodies against TNFAIP2 (1:100 dilution) or E-cadherin (1:100 dilution) were applied at room temperature for $1 \mathrm{~h}$. After washing with PBS, they were incubated with secondary antibodies against rabbit and mouse IgG conjugated to a peroxidaselabeled dextran polymer (Nichirei, Tokyo, Japan) for $1 \mathrm{~h}$. Color was developed with 3,3'-diaminobenzidine (DAB) in $50 \mathrm{mM}$ Tris-HCL (pH 5.5) containing $0.005 \%$ hydrogen peroxidase. Sections were counterstained with hematoxylin. Images were acquired using a fluorescence microscope (IX8, Olympus). The expression of E-cadherin in tumor cells was scored as the average of detectable immunoreactions from 0 to 10 ( 0 , no staining; 10 , strong staining), in $3-5$ representative areas at $\times 20$ magnification. The expression of TNFAIP2 in tumor cells was scored as the average of detectable immunoreactions from 0 to 3 ( 0 , no staining; 1 , slight staining; 2 , medium staining; 3 , strong staining), in 3-5 representative areas at $\times 20$ magnification. Three investigators blinded to patient data independently evaluated immunoreactivity, and mean E-cadherin and TNFAIP2 scores were calculated in each individual cohort. 
The TNFAIP2 expression cut off point of 2.0 was set to the upper quartile score of the study population, and this cut off point was used in the tissue array section analysis.

\section{In situ hybridization}

The RNA scope 2.0 assay was performed following the manufacturer's instructions (Advanced Cell Diagnostics, Hayward, CA) using a proprietary probe capable of detecting TNFAIP2 [16]. Formalin-fixed paraffin-embedded sections were deparaffinized. Tissues were then treated serially with pretreatment 1 solution (endogenous peroxidase block with pretreat 1 solution at room temperature for $10 \mathrm{~min})$; pretreatment $2\left(100^{\circ} \mathrm{C}, 15 \mathrm{~min}\right.$ immersion in pretreat 2 solution); and pretreatment 3 (protease digestion, $40{ }^{\circ} \mathrm{C}$ for $30 \mathrm{~min}$ ); rinses with water were performed after each pretreatment step. Tissues were then hybridized in TNFAIP2 hybridization solution, without a cover slip, at $40{ }^{\circ} \mathrm{C}$ for $2 \mathrm{~h}$ in HybEZ Oven (Advanced Cell Diagnostics). After wash buffer steps, signal amplification from the hybridized probe was performed by the serial application of Amp 1 (preamplifier step), Amp 2 (signal enhancer step), Amp 3 (amplifier step), Amp 4 (label probe step), Amp 5, and Amp 6 (signal amplification steps); wash buffer steps were performed after each Amp step. TNFAIP2 activity was then demonstrated by the application of DAB at room temperature for $5 \mathrm{~min}$. Sections were then counterstained with hematoxylin and dehydrated through graded ethanol and xylene. Images were acquired using a fluorescence microscope (IX8, Olympus). The expression of TNFAIP2 mRNA in tumor cells was semiquantitatively scored from 0 to 3 based on the number of dots per cells in 3 representative areas at $\times 20$ magnification. Three investigators blinded to patient data performed independent evaluations, and the mean TNFAIP2 mRNA score was calculated in each individual cohort.

\section{Microarray gene expression analysis}

Gene expression profiles were assessed using the Affymetrix GeneChip Human Gene 2.0 ST array according to the manufacturer's instructions. After generating single-stranded cDNA, fragmentation and sense-strand cDNA labeling was performed with the Affymetrix GeneChip WT Terminal Labeling Kit (Affymetrix Inc., Santa Clara, CA) according to the manufacturer's protocol. After hybridization, GeneChip Fluidics Station 450 (Affymetrix Inc.) was used to wash the arrays, and scanning was performed with GeneChip Scanner 30007 G (Affymetrix Inc.). Raw intensity data from the scanned images of microarrays were preprocessed using Affymetrix Expression Console software. Expression intensities were stored as cell intensity (CEL) files, and CEL files were normalized by the robust multichip average method. These datasets were filtered, and genes with an absolute fold change greater than 2 or less than 0.5 were identified as being differentially expressed. This microarray data set has been approved by the Gene Expression Omnibus (GEO) (http://www.ncbi.nlm.gov/geo/); its accession number is GSE118233.

\section{Statistical analysis}

In human studies, samples were randomly collected and no statistical method was used to predetermine sample group sizes. All data were presented as means \pm standard error (SE), medians, and interquartile range (IQR) for continuous variables, and frequencies with percentages for categorical variables. Variables between groups were compared using the two-tailed Student's $t$-test, paired $t$-test, and Mann-Whitney $U$-test, where appropriate. Survival curves were estimated using the Kaplan-Meier method and compared using the log-rank test. Differences among groups were regarded as significant when $P<0.05$. All analyses were performed using the SPSS Version 22.0 statistical software package (IBM, Armonk, NY) and JMP version 13.0 (SAS Institute Inc., Cary, NC).

\section{Results}

\section{Acquisition of platinum resistance and upregulated TNFAIP2 expression}

Changes in global gene expression patterns between parental platinum-naïve cancer cells and derived platinumresistant cancer cells are available (the accession number of the GEO is GSE58624 (http://www.ncbi.nlm.gov/geo/). Forty-nine genes with altered expression profiles were identified in UC cells before and after the acquisition of platinum resistance [11], with higher levels of TNFAIP2 being observed in platinum-resistant 5637PR cells than in platinum-naïve 5637 cells. Consistent with global gene expression data, we verified stronger TNFAIP2 expression in platinum-resistant 5637PR cells at the protein (Fig. 1a) and transcription (Fig. 1b) levels than in platinum-naïve 5637 cells. Immunofluorescence signals from 5637PR cells also revealed a high intensity of TNFAIP2 in the cytoplasm (Fig. 1c).

\section{TNFAIP2 expression and outcomes}

To assess the clinical significance of upregulated TNFAIP2 expression in cancer, we examined the relationship between TNFAIP2 expression and the outcomes of 156 patients of urothelial cancer in the upper urinary tract treated surgically (Supplementary Table 1). The results obtained showed that 
a

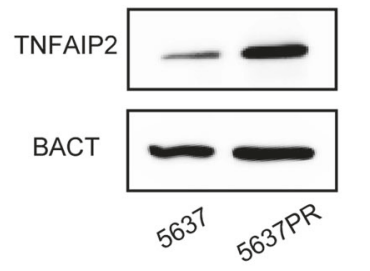

b

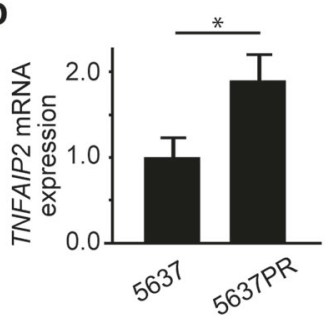

c
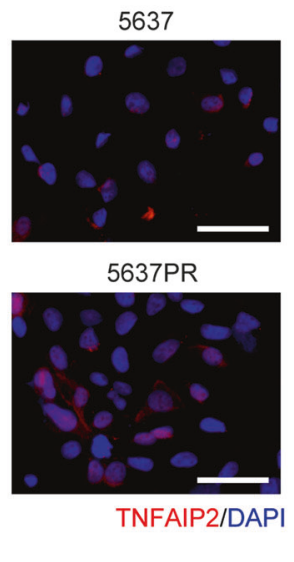

d

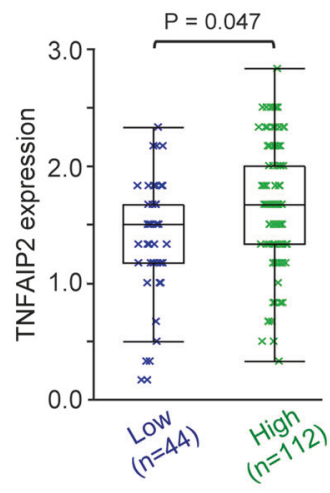

Tumor grade e

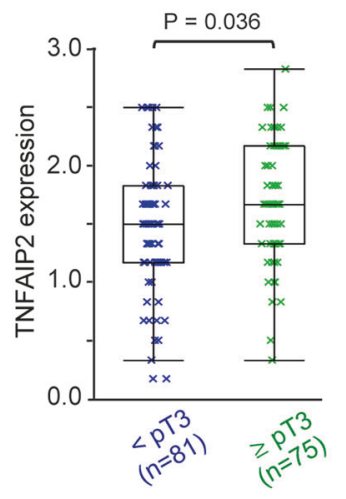

Pathological T stage

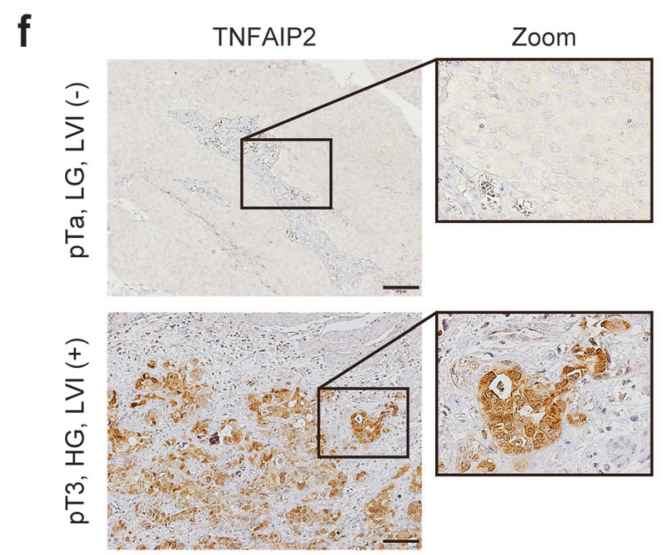

g

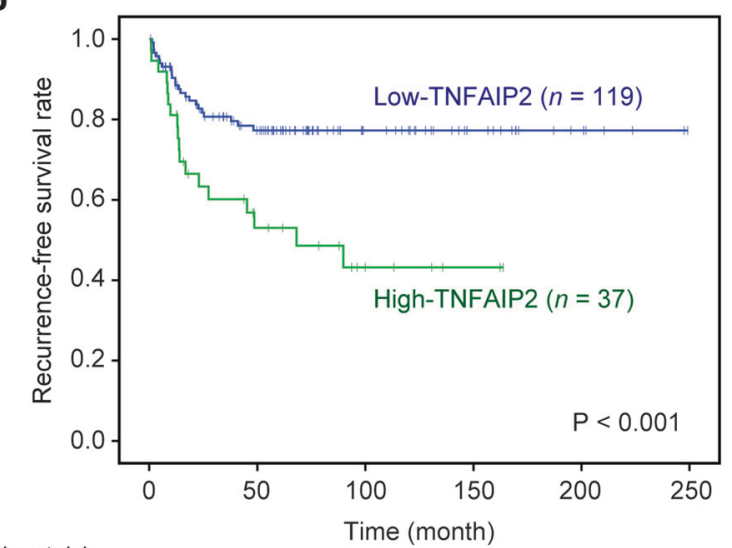

No. at risk

$\begin{array}{rrrrrrr}\text { Low } & 119 & 65 & 29 & 15 & 6 & 0 \\ \text { High } & 37 & 14 & 5 & 2 & 0 & 0\end{array}$

Fig. 1 Development of acquired platinum resistance, TNFAIP2 expression, and outcomes. a WB analysis of TNFAIP2 in platinumnaïve 5637 and platinum-resistance 5637PR cells. b mRNA expression of TNFAIP2 in 5637 and 5637PR cells. c Immunofluorescence staining for TNFAIP2 in 5637 and 5637PR cells. Bar, $100 \mu \mathrm{m}$. ${ }^{*} P<0.05$, from the Student's $t$-test. Box plots show a significant difference in TNFAIP 2 expression between low grade $(n=44)$ vs high grade $(n=112)(\mathbf{d})$, and $<\mathrm{pT} 3(n=81)$ vs $\geq \mathrm{pT} 3(n=75)$ patients $(\mathbf{e})$. h

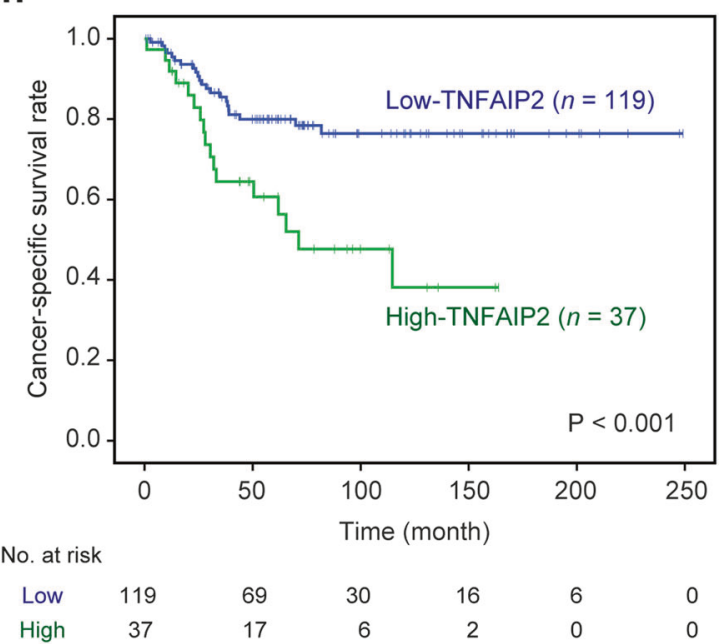

$P$ values from the Mann-Whitney $U$-test. f Representative images of TNFAIP2 expression from pTa, low-grade (LG), and lymphovascular invasion (LVI)-negative superficial tumors and pT3, high-grade (HG), LVI-positive muscle-invasive tumors. Bar, $100 \mu \mathrm{m}$. Kaplan-Meier curves according to TNFAIP2 expression of the recurrence-free (g) and cancer-specific survival (h) of 156 patients with upper urinary tract urothelial cancer following surgery. $P$ values from the logrank test 
TNFAIP2 expression was significantly upregulated in high grade $(P=0.047$, Fig. 1d) and advanced stage $(P=0.036$, Fig. 1e) tumors, which both have a major impact on the outcome of urothelial cancer [1]. Figure if shows representative images of TNFAIP2 expression from low-grade/stage lymphovascular invasion (LVI)-negative superficial tumors and high-grade/-stage LVI-positive muscle-invasive tumors. TNFAIP2 staining was stronger in muscle-invasive cancers, while weak staining was observed in superficial diseases.

During the median follow-up period of 56 months (IQR: 25-98), Kaplan-Meier curves revealed that upregulated TNFAIP2 expression was a risk factor for disease recurrence $(P<0.001$, Fig. $1 \mathrm{~g})$ as well as cancer-specific mortality $(P<0.001$, Fig. 1h). Five-year recurrence-free and cancer-specific survival rates were $53 \%$ and $61 \%$ in patients with upregulated TNFAIP2 expression, and $77 \%$ and $80 \%$ in those with down-regulated expression, respectively. In the subgroup analysis adjusted by patient tumor stages, Kaplan-Meier curves also revealed that the upregulated expression of TNFAIP2 was a risk factor for disease recurrence in patients with pT3 or greater $(p=0.046)$ and less than pT3 UTUC ( $p=0.018$ ) (Supplementary Fig. 1).

Next, we examined the significance of TNFAIP2 expression using a validated data set of 119 different patients of upper urinary tract urothelial cancer from tissue array sections (Fig. 2a-c, Supplementary Table 2). The results of Kaplan-Meier curves were similar, i.e., the upregulated expression of TNFAIP2 may reduce overall survival following surgery $(P=0.082$, Fig. $2 \mathrm{~d})$. The significance of TNFAIP2 was then examined using a different cohort of 54 patients with urinary bladder cancer who underwent radical cystectomy without neoadjuvant CDDPbased chemotherapy (Supplementary Table 3), and the results of Kaplan-Meier curves also revealed that the upregulated expression of TNFAIP2 reduced recurrencefree $(P=0.046)$ and cancer-specific survival $(P=0.018$, Supplementary Fig. 2) following radical cystectomy.

\section{TNFAIP2 induces EMT and urothelial cancer migration}

To investigate the relationship between TNFAIP2 and EMT, we initially examined the effects of the knockdown of TNFAIP2. We transfected platinum-resistant 5637PR cells with TNFAIP2-specific siRNA (si-TNFAIP2 A and B) and evaluated subsequent changes in EMT. Real-time PCR results showed that si-TNFAIP2 reduced TNFAIP2 expression levels to lower than those in mock-transfected 5637PR cells. Furthermore, si-TNFAIP2 significantly upregulated the expression of the epithelial marker E-cadherin and downregulated the expression of the mesenchymal markers SLUG, ZEB1, and TWIST1 in 5637PR cells (Fig. 3a), a

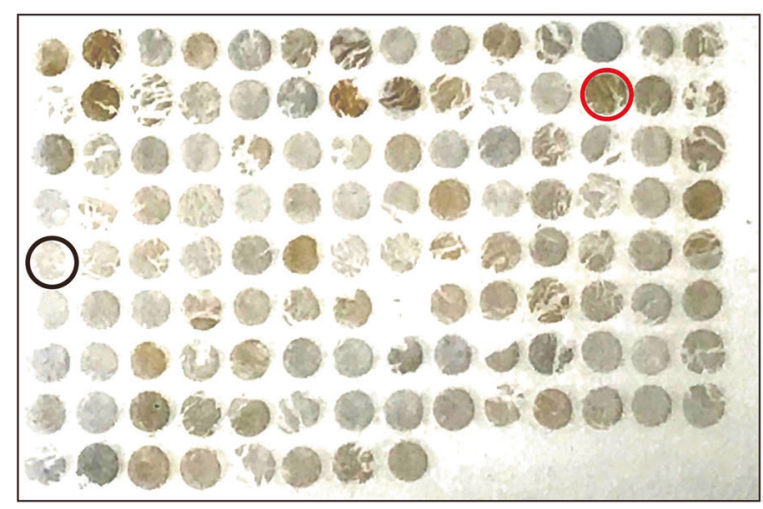

b
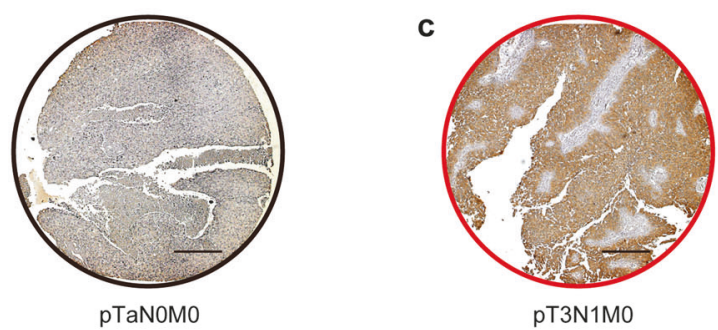

d

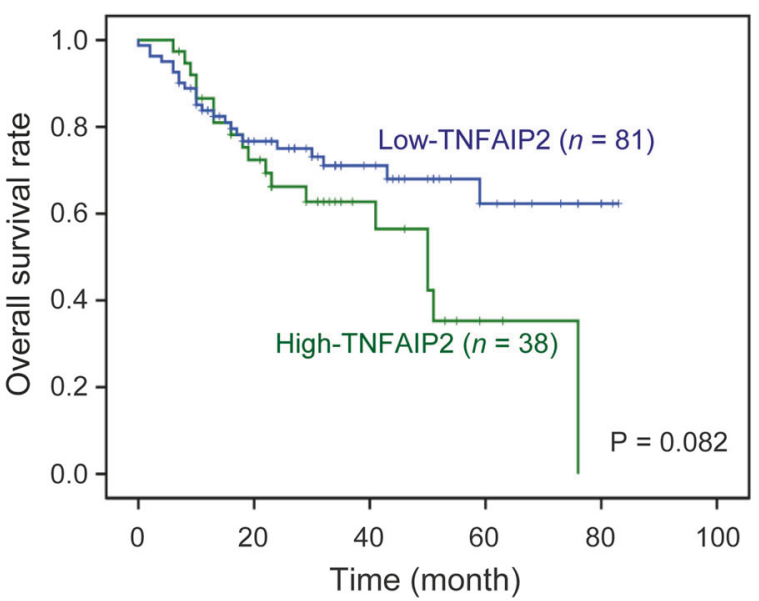

No. at risk

$\begin{array}{lrrrrrr}\text { Low } & 81 & 49 & 24 & 10 & 4 & 0 \\ \text { High } & 38 & 25 & 10 & 2 & 0 & 0\end{array}$

Fig. 2 External validation for TNFAIP2 expression on clinical outcomes using a tissue array section. a Upper urinary tract urothelial cancer tissue array containing 119 cases of upper urinary tract urothelial cancer. Representative images of weak (b) and strong (c) TNFAIP2 expression. Bar, $250 \mu \mathrm{m}$. d Kaplan-Meier curves according to TNFAIP2 expression of the overall survival of 119 patients comprising this tissue array. $P$ values from the log-rank test

revealing that the knockdown of TNFAIP2 negatively regulated EMT in platinum-resistant cancer cells. Regarding motile function, a Matrigel invasion assay showed that 5637PR cells transfected with si-TNFAIP2 exhibited significantly reduced invasion activity (Fig. 3b). Furthermore, real-time PCR results on the effects of TNFAIP2 overexpression in 5637 cells revealed downregulated 


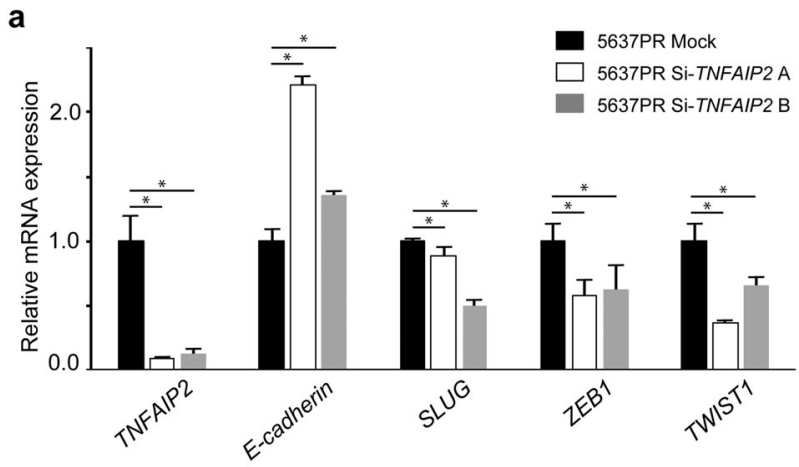

b

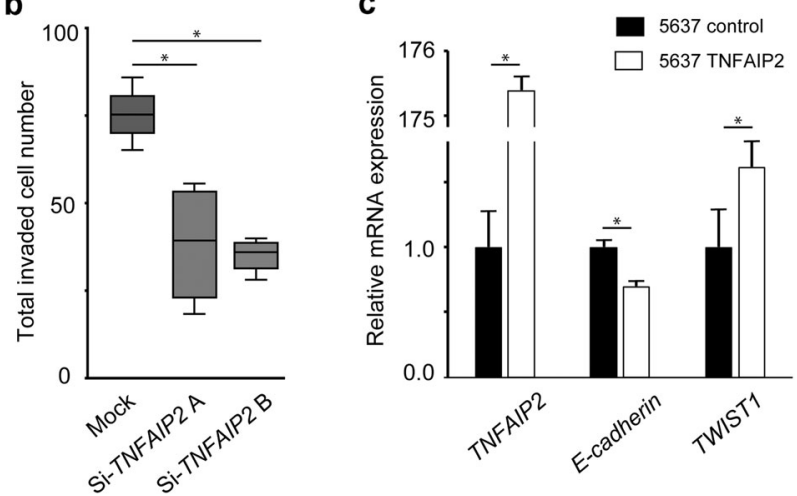

Fig. 3 Knockdown and overexpression of TNFAIP2 and urothelial cancer migration. a Effects of TNFAIP2 knockdown and EMT in platinum-resistant 5637PR cells. b Matrigel invasion assay of 5637PR cells transfected with si-TNFAIP2 $(n=4)$. Box-and-whisker plots show medians (line within box), upper and lower quartiles (bounds of box), and minimum and maximum values (bars). c Effects of TNFAIP2 overexpression and EMT on platinum-naïve 5637 cells. $* P<0.05$, from Student's $t$-test

E-cadherin expression and upregulated TWISTI expression after the transfection of TNFAIP2 (Fig. 3c), suggesting that TNFAIP2 overexpression positively regulated EMT in platinum-naïve cancer cells via TWIST1 expression.

\section{Clinical relevance of EMT and increased TNFAIP2 expression after the platinum treatment}

We then examined whether CDDP affected EMT and TNFAIP2 expression within UC tumors from humans. In order to achieve this, we investigated changes in EMT and TNFAIP2 expression between 16 matched pre- and postCDDP-treated sections of urinary bladder UC (Supplementary Table 4). The hallmark of EMT is the loss of E-cadherin expression [17], and immunostaining of 16 tumor sections revealed a significantly lower expression level of E-cadherin (Fig. 4a, $P<0.001$ ) in residual UC cells after CDDP. In contrast, upregulated TNFAIP2 expression was confirmed in residual UC cells after CDDP (Fig. $4 \mathrm{~b}, P=0.053$ ) and 11 cohorts exhibited stronger TNFAIP2 expression with a median increase of $60 \%$. We further elucidated the relationship between E-cadherin and TNFAIP2 expression using a schematic dynamics of matched bladder urothelial cancer sections obtained before and after CDDP-based chemotherapy. Figure $4 \mathrm{c}$ shows that E-cadherin expression sequentially decreased, while TNFAIP2 expression increased when tumors were treated with CDDP-based chemotherapy. These results were followed at the transcription level, showing that a higher TNFAIP2 mRNA level was detected in residual UC cells after CDDP (Fig. 4d, $P=$ 0.062 ) by in situ hybridization.

\section{MTDH expression mediates TNFAIP2-derived EMT acquisition in urothelial cancer}

To investigate the relationship between TNFAIP2 and EMT, we attempted to examine changes in global gene expression after the knockdown of TNFAIP2 (Fig. 5a). In a comparison of 5637PR cells with and without the knockdown of TNFAIP2, the expression of 19 genes was altered after siTNFAIP2 transfection (11 downregulated and 8 upregulated genes) (Table 1). Among these 19 genes, we focused on the expression of metadherin (for metastasis adhesion protein, $\mathrm{MTDH}$ ), also known as astrocyte elevated gene-1 protein (AEG-1) or lysine-rich CEACAM1 co-isolated (LYRIC), which has been suggested to function as an EMT regulator [18]. We hypothesized that changes in EMT and the migratory potential of platinum-resistant cancer cells are affected by TNFAIP2 via MTDH expression.

Real-time PCR results showed that platinum-resistant 5637PR cells had significantly higher mRNA levels of $M T D H$ than platinum-naïve 5637 cells (Fig. 5b). Furthermore, 5637PR cells transfected with si-TNFAIP2 had lower $M T D H$ expression levels than mock-transfected cells (Fig. 5c), indicating a strong relationship between TNFAIP2 and MTDH expression in cancers cells after the acquisition of platinum resistance. To deepen our understanding of MTDH and EMT, we then transfected 5637PR cells with $M T D H$-specific siRNA (si-MTDH A and B) and evaluated subsequent changes in EMT. Real-time PCR results showed that si-MTDH upregulated E-cadherin expression and downregulated TWIST1 expression (Fig. 5d), which is consistent with the results shown in Fig. 3. The present results indicated that si-MTDH slightly upregulated the expression of TNFIP2 in 5637PR cells (Supplementary Fig. 3). A Matrigel invasion assay revealed significant decreases in motile function after the knockdown of MTDH in 5637PR cells (Fig. 5e).

We further validated this TNFAIP2-MTDH axis on EMT using two other human bladder cancer cell lines, T24 and UMUC-3. Real-time PCR results showed that si-TNFAIP2 $B$ reduced TNFAIP2 expression levels to lower than those in mock-transfected cell lines. The knockdown of TNFAIP2 expression by si-TNFAIP2 B significantly downregulated 
Fig. 4 EMT and TNFAIP2 expression in 16 clinical samples of bladder urothelial cancer periplatinum treatment.

Immunostaining of E-cadherin $\mathbf{a}$ and TNFAIP2 $\mathbf{b}$ expression in matched bladder urothelial cancer sections obtained before and after CDDP-based chemotherapy. Panels a and b show spaghetti plots of responses on individual immunosignals perichemotherapy (red, decreased; green, increased). c The schematic dynamics of Ecadherin and TNFAIP2 expression in matched bladder urothelial cancer sections obtained before and after CDDP-based chemotherapy (green, pre chemotherapy; red, post chemotherapy). d Representative images of TNFAIP2 mRNA expression in a bladder urothelial cancer section obtained after CDDPbased chemotherapy. Each dot shows a single RNA transcript. Spaghetti plots in the right panel show changes in TNFAIP2 mRNA levels peri-

chemotherapy (red, decreased; orange, no change; green,

increased). $P$ values from the paired $t$-test a

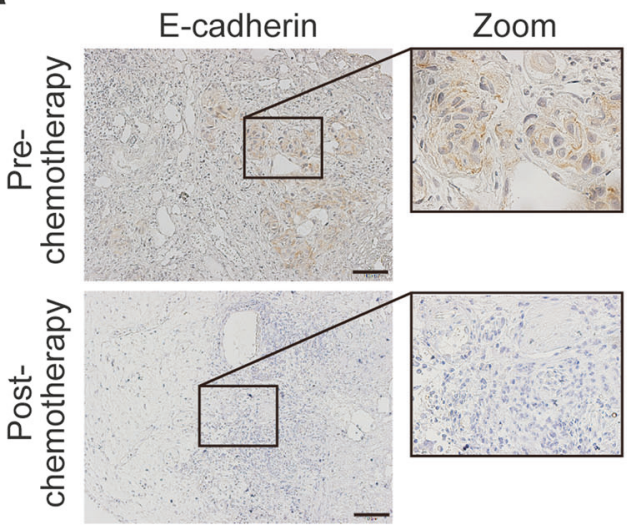

b

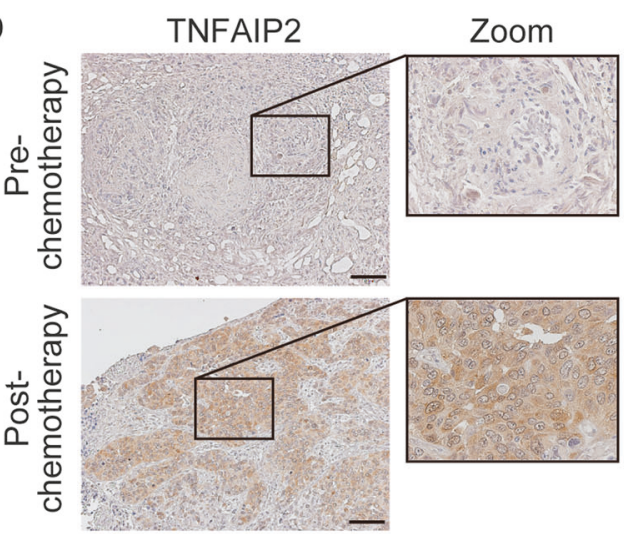

C

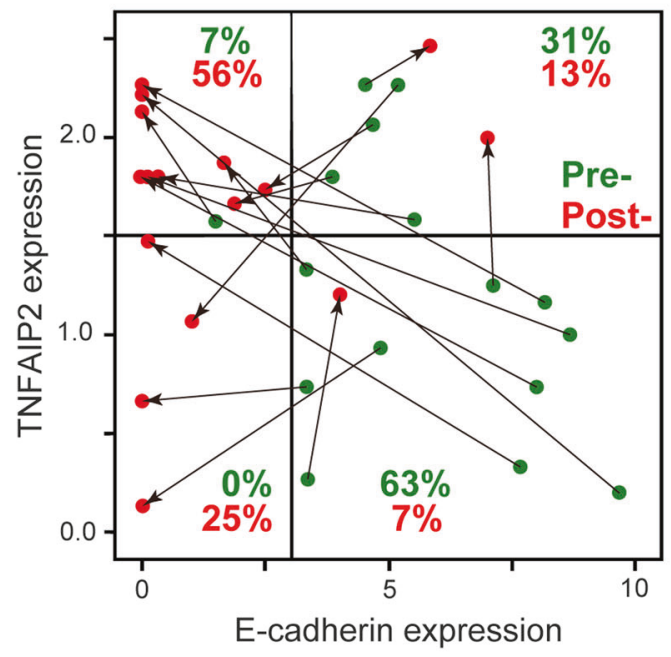

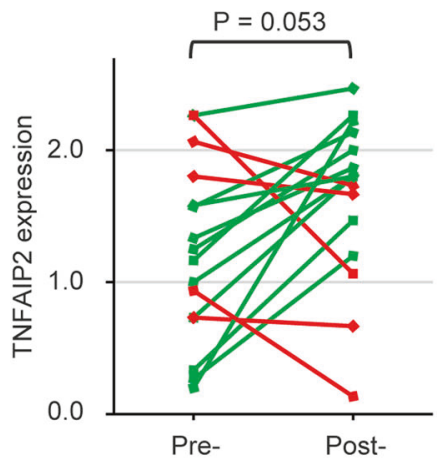

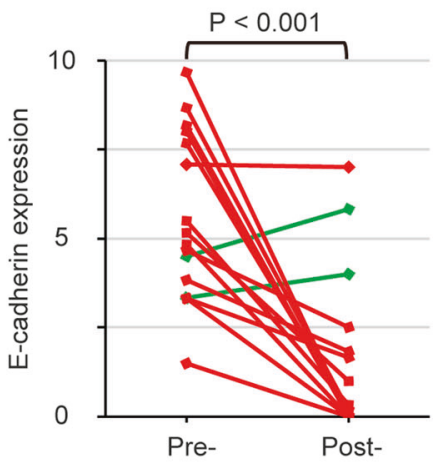

d
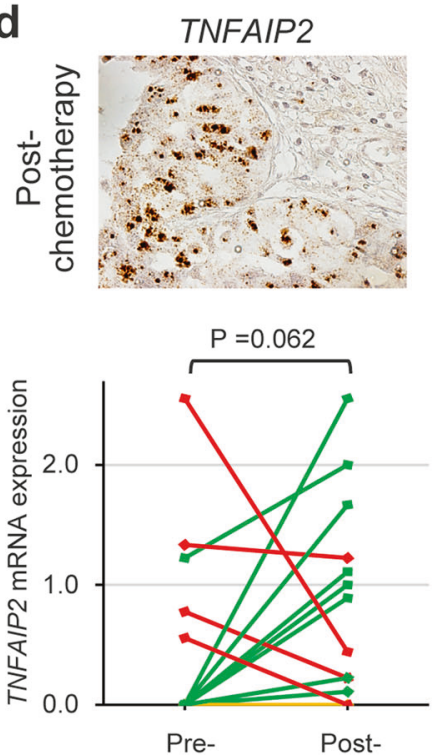

the expression of MTDH and TWIST1 in T24 (Supplementary Fig. 4a) and UMUC-3 cells (Supplementary Fig. 4b). Furthermore, the knockdown of MTDH expression by si- $M T D H$ A significantly downregulated the expression of TWIST1 in T24 (Supplementary Fig. 4c) and UMUC-3 cells (Supplementary Fig. 4d).
Collectively, the present results suggest a mechanism of TNFAIP2-derived EMT acquisition in cancers, in which MTDH expression levels in cancer cells were vital for promoting TNFAIP2-derived EMT acquisition. Furthermore, TNFAIP2 expression levels may be negatively regulated by MTDH. 
a

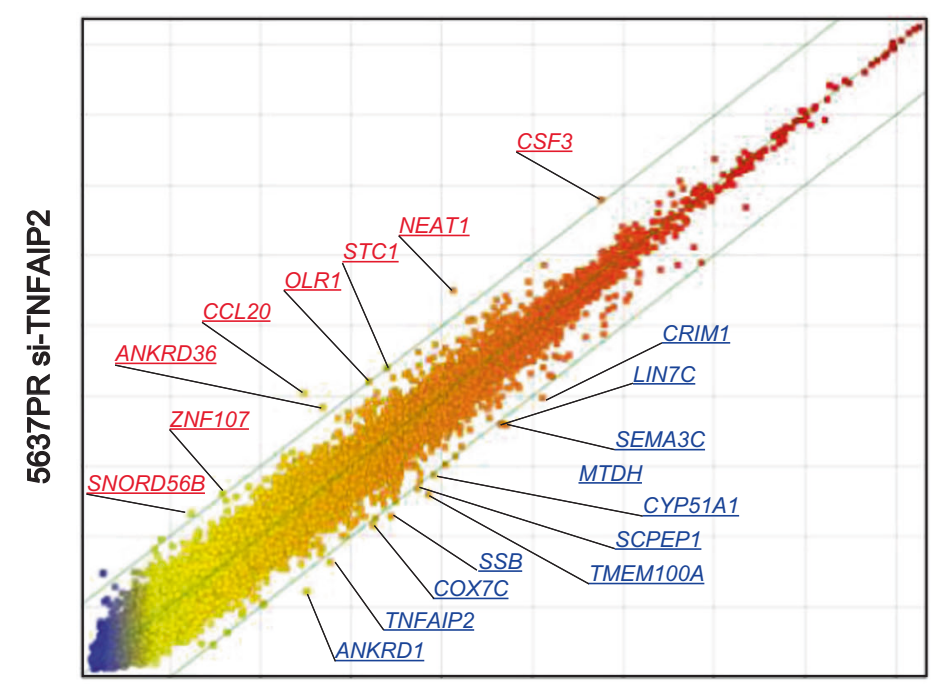

5637PR Mock

d

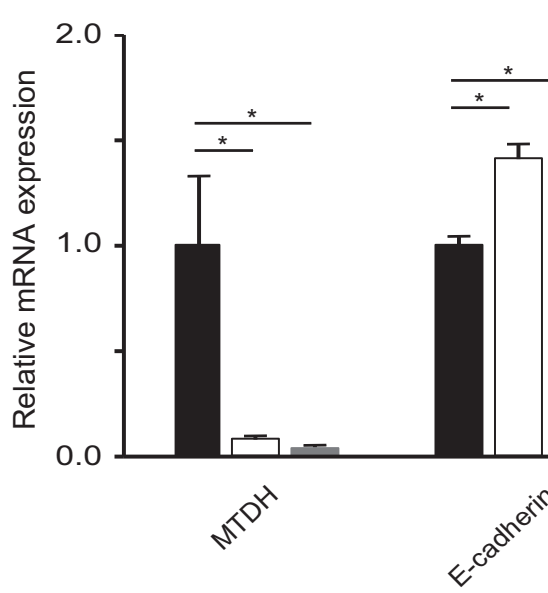

Fig. 5 Identification of MTDH as a mediator of TNFAIP2-derived EMT acquisition. a Microarray gene expression analysis revealed 19 genes (11 downregulated [blue] and 8 upregulated genes [red]) in 5637PR cells whose expression profiles were altered after si-TNFAIP2 transfection. Genes with an absolute change $>2$ or $<0.5$ from mocktransfected cells were identified as being differentially expressed. b mRNA expression of $M T D H$ in 5637 and 5637PR cells. c Effects of

\section{Discussion}

In this study, we analyzed the role of TNFAIP2 in EMT for human UC cells. The results obtained demonstrated that upregulated TNFAIP2 expression was prognostic and associated with high tumor grades and advanced tumor stages clinically. Also, upregulated TNFAIP2 expression is a positive regulator of EMT after the acquisition of platinum resistance and subsequent tumor migration. Based on global gene expression profiles using the knockdown of TNFAIP2 dent's $t$-test
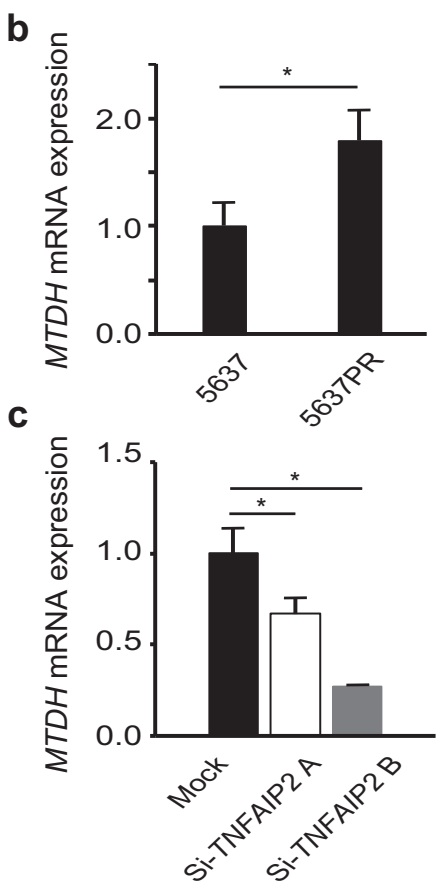

e

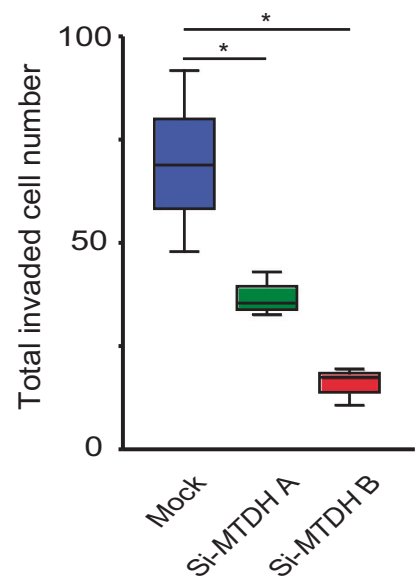

TNFAIP2 knockdown and MTDH expression on 5637PR cells. d Effects of MTDH knockdown and E-cadherin and TWIST1 expression on 5637PR cells. e Matrigel invasion assay of 5637PR cells transfected with si-MTDH $(n=4)$. Box-and-whisker plots show medians (line within box), upper and lower quartiles (bounds of box), and minimum and maximum values (bars). ${ }^{*} P<0.05$, from the Stu-

in platinum-resistant 5637PR cells, we further identified MTDH as a downstream target of TNFAIP2-derived EMT in platinum-resistant tumors.

TNFAIP2 was initially described as a gene whose expression may be induced by TNF-alpha in human umbilical vein endothelial cells [19]. In the context of cancer, TNFAIP2 expression was shown to be induced by retinoic acid in cells that express a fusion oncoprotein (promyelocytic leukemia-retinoic acid receptor alpha), which suggested that TNFAIP2 is a target gene of retinoic 
Table 1 A list of 19 genes that were differentially expressed in 5637 PR cells after si-TNFAIP2 transfection

\begin{tabular}{|c|c|c|}
\hline Gene name & Gene symbol & Fold change \\
\hline \multicolumn{3}{|l|}{ Downregulated } \\
\hline$A N K R D 1$ & Ankyrin repeat domain 1 & 0.19 \\
\hline MEM200A & Transmembrane protein $200 \mathrm{~A}$ & 0.20 \\
\hline$S S B$ & Sjogren syndrome antigen B & 0.22 \\
\hline CRIMI & $\begin{array}{l}\text { Cysteine rich transmembrane BMP } \\
\text { regulator } 1\end{array}$ & 0.22 \\
\hline TNFAIP2 & $\begin{array}{l}\text { Tumor necrosis factor alpha-induced } \\
\text { protein } 2\end{array}$ & 0.22 \\
\hline$M T D H$ & Metadherin & 0.22 \\
\hline COX7 C & Cytochrome $\mathrm{c}$ oxidase subunit $7 \mathrm{C}$ & 0.23 \\
\hline SEMA3C & Semaphorin C & 0.24 \\
\hline LIN7C & Lin-7 homolog $\mathrm{C}$ & 0.24 \\
\hline SCPEP1 & Serine carboxypeptidase 1 & 0.24 \\
\hline CYP51A1 & $\begin{array}{l}\text { Cytochrome P450 family } \\
51 \text { subfamily A member } 1\end{array}$ & 0.25 \\
\hline \multicolumn{3}{|l|}{ Upregulated } \\
\hline CCL2O & $\mathrm{C}-\mathrm{C}$ motif chemokine ligand 20 & 7.89 \\
\hline NEAT1 & $\begin{array}{l}\text { Nuclear paraspeckle assembly } \\
\text { transcript } 1\end{array}$ & 6.23 \\
\hline$A N K R D 1$ & Ankyrin repeat domain 36 & 4.72 \\
\hline SNORD56B & Small nucleolar RNA, C/D box 56B & 4.44 \\
\hline CSF3 & Colony stimulating factor 3 & 4.20 \\
\hline ZNF107 & Zinc finger protein 107 & 4.17 \\
\hline OLR1 & $\begin{array}{l}\text { Oxidized low density lipoprotein } \\
\text { receptor } 1\end{array}$ & 4.08 \\
\hline STC1 & Stanniocalcin 1 & 4.00 \\
\hline
\end{tabular}

acid in acute promyelocytic leukemia [20]. TNFAIP2 expression was shown to be associated with cancer progression in nasopharyngeal carcinoma [12, 21], breast cancer [13], and esophageal carcinoma [14]. Chen et al. reported that the upregulated expression of TNFAIP2 by Epstein-Barr virus oncoprotein latent membrane protein 1 through NF-kappa B promoted cancer cell motility in nasopharyngeal carcinoma [21]. We herein revealed that the clinical significance of upregulated TNFAIP2 expression in cancer, revealing that upregulated TNFAIP2 expression may be prognostic and reduce overall survival following surgery in patients with urinary UC.

We then focused on the role of TNFAIP2 as a positive regulator of EMT in UC cells after the acquisition of platinum resistance. We revealed that the downregulated expression of TNFAIP2 by si-RNA resulted in the upregulated expression of E-cadherin and downregulated expression of SLUG, ZEB1, and TWIST1, leading to decreased migratory potential in UC cells after the acquisition of platinum resistance. The overexpression of TNFAIP2 downregulated E-cadherin expression and upregulated TWIST1 expression. We clinically demonstrated changes in TNFAIP2 expression using 16 matched pre- and post-CDDP-treated UC sections and revealed increased TNFAIP2 and decreased E-cadherin expression levels after CDDP. TNFAIP2 expression in UC samples was verified at the transcription level, showing that higher TNFAIP2 mRNA levels were detected in tumor cells after CDDP by in situ hybridization. The RNA scope, a new RNA in situ hybridization system using target-specific signals, enables the quantitative detection of mRNA in formalin-fixed paraffin-embedded tissue samples [16].

We further investigated the regulatory molecules connecting TNFAIP2 and EMT. A comparison of global gene expression with and without TNFAIP2 knockdown revealed MTHD as a candidate gene that regulates EMT-like changes in UC cells. MTDH, also known as AEG-1 or LYRIC, was initially identified as a TNF-alpha-induced gene in primary human fetal astrocytes infected with human immunodeficiency type 1 [22]. Brown et al. subsequently isolated a domain in the protein MTDH from phage expression libraries of breast carcinoma cDNA. They showed that MTDH is a protein overexpressed in breast cancers and mediates the lung-specific dissemination of metastatic cells [23]. MTDH expression has been associated with cancer metastasis and chemoresistance [18, 24], and has also been suggested to promote cancer invasion or metastasis through the induction of EMT [18, 25-28]. Liang et al. reported that MTDH drives the expression of cancer stem-like cells, a cell subpopulation responsible for chemoresistance, through TWIST1 expression indirectly facilitated by histone H3 acetylation on the TWIST1 promoter [29]. Our results revealed that the downregulation of MTDH by si-RNA upregulated E-cadherin expression and downregulated TWISTI expression, leading to decreased migratory potential in UC cells after the acquisition of platinum resistance. These results suggest that MTDH is a target of TNFAIP2 for the development of EMT. Although further investigations are needed to clarify the precise interaction between the TNFAIP2/MTDH axis and EMT in malignancies, our results suggest a new axis of TNFAIP2/MTDH/TWIST1 that is involved in EMT in platinum-resistant UCs and has potential as a therapeutic target in patients with cancer receiving CDDP.

This study has several limitations including the small number of clinical samples, the retrospective nature of this cohort, and heterogeneous groups of patients. We did not repeat the microarray analysis in our in vitro study. Furthermore, it remains unclear whether EMT itself plays a central role in the acquisition of platinum resistance in patients with UC. For example, intratumoral heterogeneity profiles are closely associated with acquired drug resistance [30]. Using the recent technical development of single-cell RNA sequencing, we investigated the influence of CDDP on intratumoral heterogeneity in platinum-naïve 5637 cells 
and platinum-resistant 5637PR cells [31]. The findings obtained revealed that both cell lines were heterogeneous populations at the single-cell level. However, we found a small fraction of the subclones of parental 5637 cells that matched 5637PR cells at the transcriptome level. These findings support platinum-resistant subclones already being present in tumors and behaving as cancer cells with acquired platinum resistance in platinum-naïve cancers. We also speculate that these subclones will dominate if tumors acquire platinum resistance and EMT features. Taken together, further investigations to more accurately elucidate the role of TNFAIP2 in EMT and associated platinum resistance are warranted.

Acknowledgements The authors would like to sincerely thank Ms Yukiko Nakajima for her assistance with the experiments. This study was supported in part by Grants-in-Aid for Scientific Research (\#15K20110 and 18K16746 to NN, \#26462429 to NT, and \#26861299 to TK) from the Ministry of Education, Culture, Sports, Science, and Technology of Japan. This work was also supported in part by a Keio University Grant-in-Aid for the Encouragement of Young Medical Scientists to NN from Keio University School of Medicine. The study was also supported in part by a research grant to NT from The YASUDA Medical Foundation, Japan, The Takeda Science Foundation, Japan, and The Uehara Memorial Foundation, Japan.

\section{Compliance with ethical standards}

Conflict of interest RM has received honoraria from Novartis and Pfizer. TK has received honoraria from Sanofi, Astellas, Takeda, Bayer, and Janssen. MO has received honoraria from Astellas, Bayer, Novartis, and Pfizer.

Publisher's note: Springer Nature remains neutral with regard to jurisdictional claims in published maps and institutional affiliations.

\section{References}

1. Rouprêt M, Babjuk M, Compérat E, Zigeuner R, Sylvester R, Burger M, et al. European association of urology guidelines on upper urinary tract urothelial cell carcinoma: 2015 update. Eur Urol. 2015;68:868-79.

2. Alfred Witjes J, Lebret T, Compérat EM, Cowan NC, De Santis M, Bruins HM, et al. Updated 2016 EAU guidelines on muscleinvasive and metastatic bladder cancer. Eur Urol. 2017;71:462-75.

3. Margulis V, Shariat SF, Matin SF, Kamat AM, Zigeuner R, Kikuchi E, et al. Outcomes of radical nephroureterectomy: a series from the upper tract urothelial carcinoma collaboration. Cancer 2009;115:1224-33.

4. Loehrer PJ Sr, Einhorn LH, Elson PJ, Crawford ED, Kuebler P, Tannock I, et al. A randomized comparison of cisplatin alone or in combination with methotrexate, vinblastine, and doxorubicin in patients with metastatic urothelial carcinoma: a cooperative group study. J Clin Oncol. 1992;10:1066-73.

5. von der Maase H, Hansen SW, Roberts JT, Dogliotti L, Oliver T, Moore MJ, et al. Gemcitabine and cisplatin versus methotrexate, vinblastine, doxorubicin, and cisplatin in advanced or metastatic bladder cancer: results of a large, randomized, multinational, multicenter, phase III study. J Clin Oncol. 2000;18:3068-77.
6. Kang Y, Massagué J. Epithelial-mesenchymal transitions: twist in development and metastasis. Cell 2004;118:277-9.

7. Lamouille S, Xu J, Derynck R. Molecular mechanisms of epithelial-mesenchymal transition. Nat Rev Mol Cell Biol. 2014;15:178-96.

8. Arumugam T, Ramachandran V, Fournier KF, Wang H, Marquis L, Abbruzzese JL, et al. Epithelial to mesenchymal transition contributes to drug resistance in pancreatic cancer. Cancer Res. 2009;69:5820-8.

9. McConkey DJ, Choi W, Marquis L, Martin F, Williams MB, Shah $\mathrm{J}$, et al. Role of epithelial-to-mesenchymal transition (EMT) in drug sensitivity and metastasis in bladder cancer. Cancer Metastasis Rev. 2009;28:335-44.

10. Tanaka N, Miyajima A, Kosaka T, Miyazaki Y, Shirotake S, Shirakawa $\mathrm{H}$, et al. Acquired platinum resistance enhances tumour angiogenesis through angiotensin II type 1 receptor in bladder cancer. Br J Cancer. 2011;105:1331-7.

11. Tanaka N, Kosaka T, Miyazaki Y, Mikami S, Niwa N, Otsuka Y, et al. Acquired platinum resistance involves epithelial to mesenchymal transition through ubiquitin ligase FBXO32 dysregulation. JCI Insight. 2016;1:e83654.

12. Chen LC, Chen CC, Liang Y, Tsang NM, Chang YS, Hsueh C. A novel role for TNFAIP2: its correlation with invasion and metastasis in nasopharyngeal carcinoma. Mod Pathol. 2011;24: $175-84$.

13. Jia L, Zhou Z, Liang H, Wu J, Shi P, Li F, et al. KLF5 promotes breast cancer proliferation, migration and invasion in part by upregulating the transcription of TNFAIP2. Oncogene. 2016;35: 2040-51.

14. Xie Y, Wang B. Downregulation of TNFAIP2 suppresses proliferation and metastasis in esophageal squamous cell carcinoma through activation of the $\mathrm{Wnt} / \beta$-catenin signaling pathway. Oncol Rep. 2017;37:2920-8.

15. Kosaka T, Kikuchi E, Mikami S, Miyajima A, Shirotake S, Ishida $\mathrm{M}$, et al. Expression of snail in upper urinary tract urothelial carcinoma: prognostic significance and implications for tumor invasion. Clin Cancer Res. 2010;16:5814-23.

16. Wang F, Flanagan J, Su N, Wang LC, Bui S, Nielson A, et al. RNAscope: a novel in situ RNA analysis platform for formalinfixed, paraffin-embedded tissues. J Mol Diagn. 2012;14:22-9.

17. Onder TT, Gupta PB, Mani SA, Yang J, Lander ES, Weinberg RA. Loss of E-cadherin promotes metastasis via multiple downstream transcriptional pathways. Cancer Res. 2008;68:3645-54.

18. Zhu K, Dai Z, Pan Q, Wang Z, Yang GH, Yu L, et al. Metadherin promotes hepatocellular carcinoma metastasis through induction of epithelial-mesenchymal transition. Clin Cancer Res. 2011;17: 7294-302.

19. Wolf FW, Sarma V, Seldin M, Drake S, Suchard SJ, Shao H, et al. B94, a primary response gene inducible by tumor necrosis factoralpha, is expressed in developing hematopoietic tissues and the sperm acrosome. J Biol Chem. 1994;269:3633-40.

20. Rusiniak ME, Yu M, Ross DT, Tolhurst EC, Slack JL. Identification of B94 (TNFAIP2) as a potential retinoic acid target gene in acute promyelocytic leukemia. Cancer Res. 2000;60:1824-9.

21. Chen CC, Liu HP, Chao M, Liang Y, Tsang NM, Huang HY, et al. NF-KB-mediated transcriptional upregulation of TNFAIP2 by the Epstein-Barr virus oncoprotein, LMP1, promotes cell motility in nasopharyngeal carcinoma. Oncogene. 2014;33:3648-59.

22. Su ZZ, Kang DC, Chen Y, Pekarskaya O, Chao W, Volsky DJ, et al. Identification and cloning of human astrocyte genes displaying elevated expression after infection with HIV-1 or exposure to HIV-1 envelope glycoprotein by rapid subtraction hybridization, RaSH. Oncogene. 2002;21:3592-602.

23. Brown DM, Rouslahti E. Metadherin, a cell surface protein in breast tumors that mediates lung metastasis. Cancer Cell. 2004; $5: 365-74$. 
24. Hu G, Chong RA, Yang Q, Wei Y, Blanco MA, Li F, et al. MTDH activation by $8 \mathrm{q} 22$ genomic gain promotes chemoresistance and metastasis of poor-prognosis breast cancer. Cancer Cell. 2009;15:9-20.

25. Li X, Kong X, Huo Q, Guo H, Yan S, Yuan C, et al. Metadherin enhances the invasiveness of breast cancer cells by inducing epithelial to mesenchymal transition. Cancer Sci. 2011;102: $1151-7$.

26. Yu C, Liu Y, Tan H, Li G, Su Z, Ren S, et al. Metadherin regulates metastasis of squamous cell carcinoma of the head and neck via AKT signalling pathway-mediated epithelial-mesenchymal transition. Cancer Lett. 2014;343:258-67.

27. Yao Y, Gu X, Liu H, Wu G, Yuan D, Yang X, et al. Metadherin regulates proliferation and metastasis via actin cytoskeletal remodelling in non-small cell lung cancer. $\mathrm{Br} \mathrm{J}$ Cancer. 2014;111:355-64.

28. Tang J, Shen L, Yang Q, Zhang C. Overexpression of metadherin mediates metastasis of osteosarcoma by regulating epithelialmesenchymal transition. Cell Prolif. 2014;47:427-34.

29. Liang Y, Hu J, Li J, Liu Y, Yu J, Zhuang X, et al. Epigenetic activation of TWIST1 by MTDH promotes cancer stem-like cell traits in breast cancer. Cancer Res. 2015;75:3672-80.

30. Dagogo-Jack I, Shaw AT. Tumour heterogeneity and resistance to cancer therapies. Nat Rev Clin Oncol. 2018;15:81-94.

31. Tanaka N, Katayama S, Reddy A, Nishimura K, Niwa N, Hongo $\mathrm{H}$, et al. Single-cell RNA-seq analysis reveals the platinum resistance gene COX7B and the surrogate marker CD63. Cancer Med. 2018;7:6193-204. 